\title{
Multi-modality endoscopic management of a sub- mucosal pancreas-perforating gastric foreign body
}
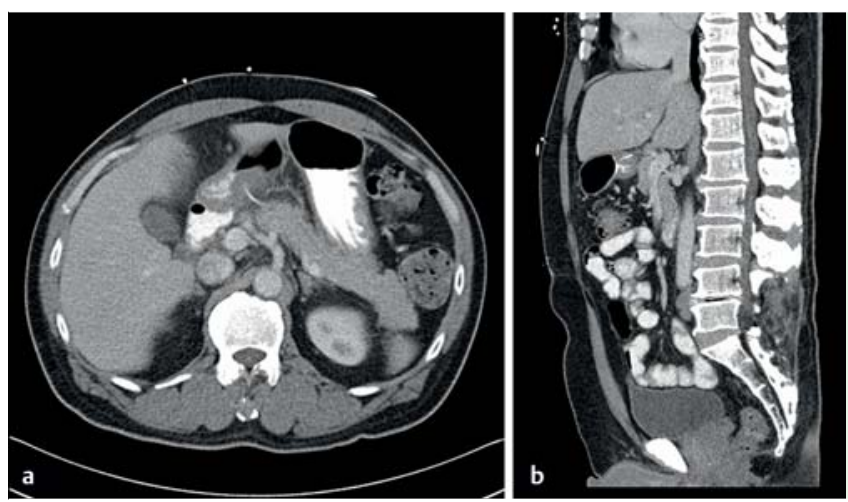

Fig. 1 Computed tomography (CT) images showing the perforating fishbone within the posterior wall of the stomach and extending towards the pancreas.
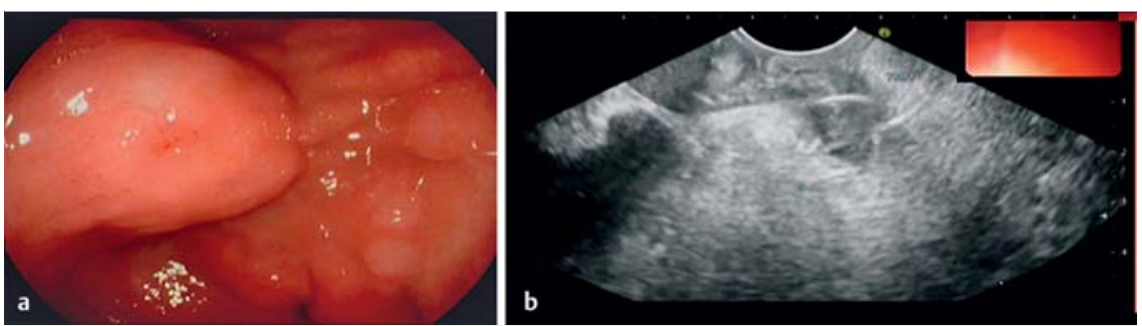

Fig. 2 The fishbone embedded in the gastric wall seen: a endoscopically; $\mathbf{b}$ on endosonographic view.
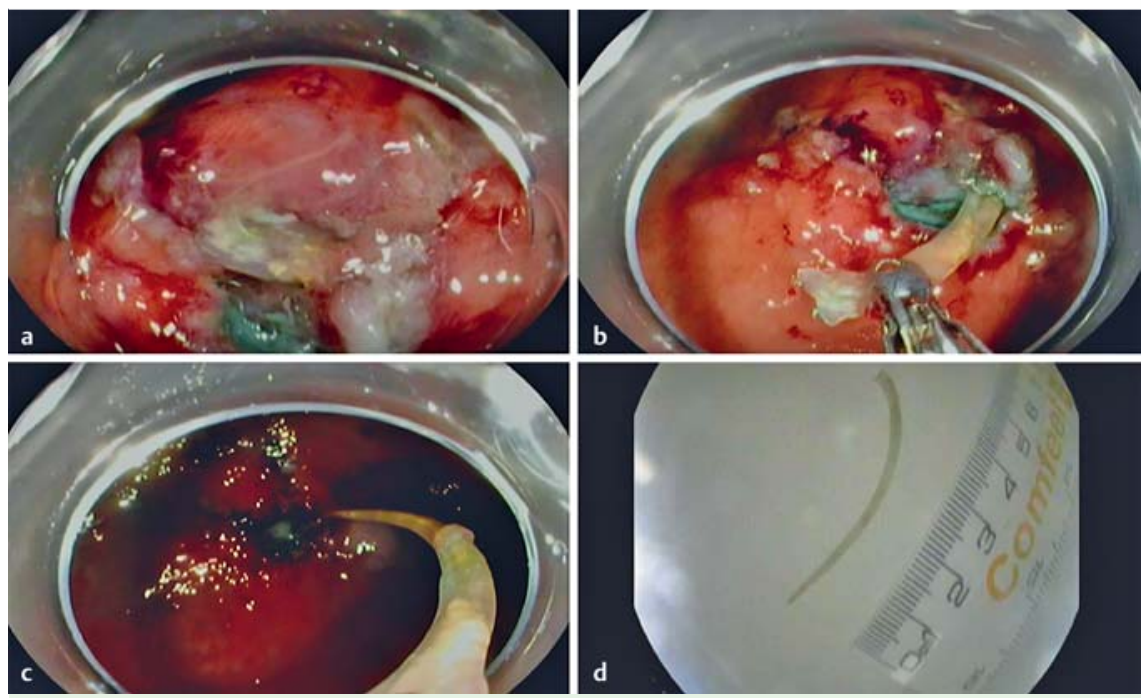

Fig. 3 The fishbone was removed using a modified endoscopic submucosal dissection (ESD) technique. a-c Endoscopic views showing the creation of a mucosal flap and extraction of the fishbone. d The extracted $5-\mathrm{cm}$ fishbone.
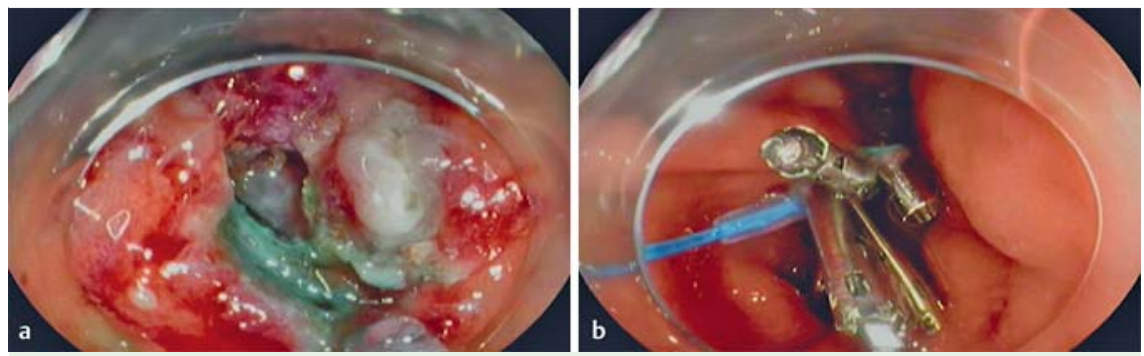

Fig. 4 Endoscopic views showing: a a large muscularis propria breach; $\mathbf{b}$ endoscopic closure of the mucosal flap.

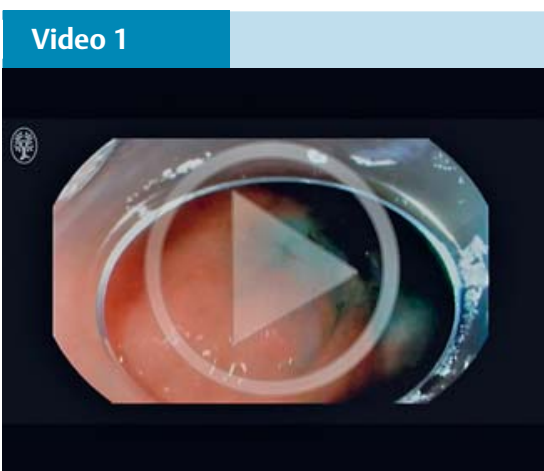

Successful removal of a fishbone embedded in the submucosa of the stomach.

A 60-year-old man presented to hospital with 3 days of epigastric pain after inadvertent ingestion of a fishbone. A computed tomography (CT) scan showed a hyperdense linear object measuring $5 \mathrm{~cm}$ that was embedded within the posterior wall of the stomach and extending towards the pancreas, in keeping with a perforating fishbone ( $\bullet$ Fig. 1). There was minimal free gas and no contrast leakage was noted on the scan. Given the location of the fishbone in the lesser sac, laparoscopic removal was thought to be technically challenging, so an attempt at endoscopic extraction was made to try to avoid him having to undergo a laparotomy.

At endoscopy ( $\bullet$ Video 1 ), no fishbone was visible in the lumen but a raised erythematous area was noted in the antrum (৫ Fig.2a). Endoscopic ultrasonography (EUS) was performed with a linear array echoendoscope to identify the hyperechoic fishbone, which extended from the submucosa into the proximal body of pancreas ( $\bullet$ Fig.2b) with no evidence of pancreatic duct injury.

Under EUS guidance, a biopsy forceps was used to mark the mucosa overlying the fishbone. A modified endoscopic submucosal dissection (ESD) technique was then used to create a mucosal flap with a hookknife (Olympus Corporation, Tokyo, Japan) after submucosal injection. The fishbone was identified after creating the mucosal flap and was grasped with a biopsy forceps and extracted ( $\bullet$ Fig. $\mathbf{3 a - c}$ ). The fishbone measured $5 \mathrm{~cm}$ in length ( $\mathbf{F i g} .3 \mathrm{~d}$ ). A large defect in the muscularis propria was seen after extraction of the fishbone 
( $\bullet$ Fig.4a). The mucosal flap was used to seal this and was closed with clips and secured with an endoloop ( $\bullet$ Fig.4b). Intraprocedural contrast injection showed no evidence of leakage.

A repeat CT scan of the abdomen the following day showed no further foreign body within the stomach and no contrast leakage, and the patient was discharged 2 days later.

Endoscopy_UCTN_Code_TTT_1AO_2AL

Competing interests: None
Christopher Lim¹, Peter Cosman ${ }^{1,2}$, Milan Bassan ${ }^{1,3}$

${ }^{1}$ Department of Upper Gastrointestinal Surgery, Liverpool Hospital, New South Wales, Australia

${ }^{2}$ University of New South Wales, Australia

${ }^{3}$ Department of Gastroenterology and Hepatology, Liverpool Hospital, New South Wales, Australia

\section{Bibliography}

Dol http://dx.doi.org/

10.1055/s-0042-117423

Endoscopy 2016; 48: E323-E324

(c) Georg Thieme Verlag KG

Stuttgart · New York

ISSN 0013-726X

\section{Corresponding author}

\section{Milan Bassan, MBBS, FRACP}

Department of Gastroenterology and Hepatology Liverpool Hospital

New South Wales

Australia

milan.bassan@gmail.com 